\title{
Reaction-Diffusion Devices using Minority-Carrier Transport in Semiconductors
}

\author{
Yuusaku Nishimiya, Tetsuya Asai, and Yoshihito Amemiya \\ Department of Electrical Engineering, Hokkaido University, Kita 13, Nishi 8, Kita-ku, Sapporo, 060-8628, Japan. \\ Phone : +81-11-706-6080, Fax : +81-11-706-7890, E-mail : asai@sapiens-ei .eng.hokudai .ac.jp
}

\section{INTRODUCTION}

The purpose of this work is the development of novel silicon devices that imitate the lively, dynamic behavior of the reactiondiffusion (RD) system. The RD system is a complex system in which reaction and diffusion of chemical species coexist under a nonequilibrium condition[1]. It produces a variety of orders, rhythms and selforganizing phenomena observed in nature and in life. We are developing electronic analogues of the RD system with the aim of creating novel information-processing hardware. As the first step toward the goal, we propose in this report a RD device that utilizes autocatalytic multiplication and diffusion of minority carriers in a $p-n-p-n$ diode array.

\section{THE RD DEVICE USING MINORITY-CARRIER TRANSPORT}

The RD device we propose is illustrated in Fig. 1. It consists of four-layer $p-n-p-n$ diodes arranged regularly on a silicon substrate. Each $p-n-p-n$ diode is connected with a capacitor and a current source to form a relaxation oscillator as shown in Fig. 2. This oscillator acts as a unit cell that imitates a chemical reaction, a substrate-depleted reaction, so we call the oscillator a reaction cell. During the cycle of oscillation, the reaction cell produces minority carriers (electrons) in the inner $p$ region. The point of our idea is to use the minority carriers as diffusion substances; i.e., electrons produced by a reaction cell will travel through the inner $\mathrm{p}$ region by diffusion and reach the neighboring reaction cells to activate the cells (see Fig. 1). A two-dimensional RD system can thus be constructed on a silicon chip.

The state of the reaction cell can be represented by two variables, i.e., charge $u$ stored on the capacitor and charge $v$ of the minority carriers in the $p-n-p-n$ diode. The dynamics of the reaction cell is described by

$$
\begin{aligned}
& \frac{d u}{d t}=i(u)-\frac{u}{\tau(u, v)} \\
& \frac{d v}{d t}=-v+\frac{u}{\tau(u, v)}
\end{aligned}
$$

where charges $u$ and $v$ are normalized. The bias current $i(u)$ from the $p$ MOS current source is a function of $u$ and is also normalized. The characteristic of the $p-n-p$ - $n$ diode is represented by nonlinear function $\tau(u, v)$. Minority-carrier charge $v$ increases through a multiplication process caused by the feedback mechanism of the $p-n-p$ - $n$ diode, while capacitor charge $u$ decreases by the amount equal to the increased minority carriers. The operation is categorized into a substrate-depleted reaction.

The reaction cell can be oscillatory (astable) or excitatory (monostable) depending on supply voltage $V_{D D}$. It is oscillatory if $V_{D D}$ is higher than breakover voltage $V_{B}$ of the $p-n-p-n$

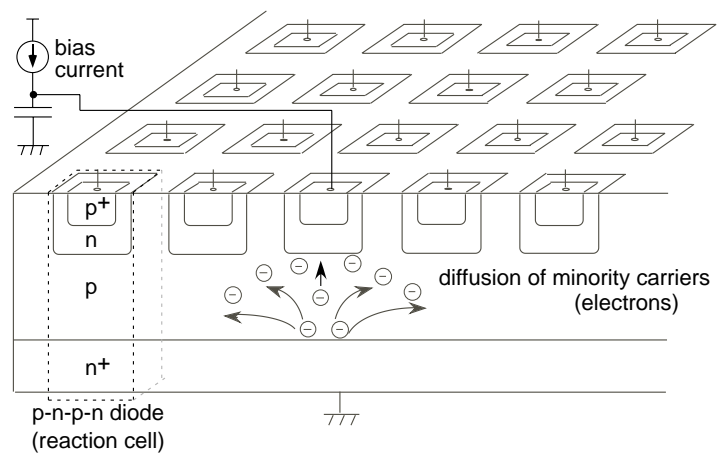

Fig. 1. The reaction-diffusion device (RD device) consisting of an array of $p$ - $n$ - $p$ - $n$ diodes.

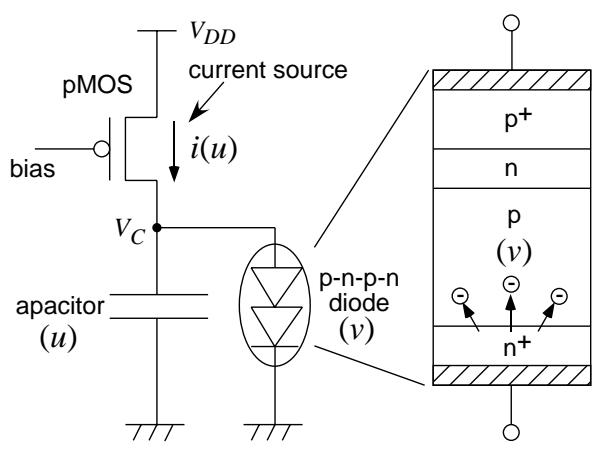

Fig. 2. The reaction cell consisting of a $p-n-p$ - $n$ diode, a capacitor, and a current source. The current source consists of a $p$ MOS transistor.

diode, and excitatory if $V_{D D}$ is lower than $V_{B}$. In the oscillatory condition $\left(V_{D D}>V_{B}\right)$, the capacitor is charged by bias current $i(u)$ and consequently, capacitor charge $u$ increases until capacitor voltage $V_{C}$ reaches breakover voltage $V_{B}$. When $V_{C}$ reaches $V_{B}$, the breakover of the $p-n-p$ - $n$ diode starts and minority carriers are injected from the $n+$ region to the $p$ region. Then, the autocatalytic multiplication of minority carriers occurs to turn the diode on. The stored charge on the capacitor flows into the diode, so capacitor charge $u$ (therefore capacitor voltage $V_{C}$ ) decreases and consequently, the diode off. The reaction cell repeats this cycle and produces oscillatory dynamics. Figures 3(a) and 3(b) illustrate an example of the numerical solutions to Eqs. (1) and (2). Figure 3(a) shows the relaxation oscillations in variables $u$ and $v$. The limit-cycle attractor is shown in Fig. 3(b).

In the excitatory condition $\left(V_{D D}<V_{B}\right)$, capacitor voltage $V_{C}$ cannot reach breakover voltage $V_{B}$ because $V_{C}$ does not exceed supply voltage $V_{D D}$ (bias current $i(u)$ becomes 0 when $V_{C}$ increases up to $\left.V_{D D}\right)$. In this condition, the $p-n-p$ - $n$ diode turns on only when minority carriers are injected from neighboring diodes. Figure 4 shows an example of the excitatory behavior 


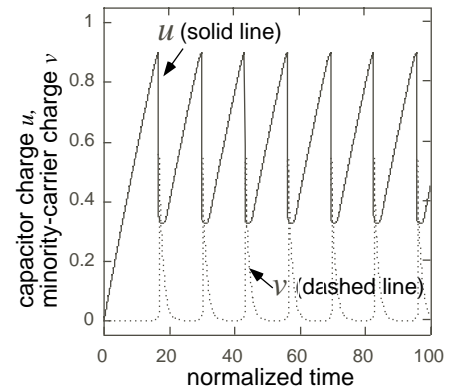

(a) time course of the unit cell circuit

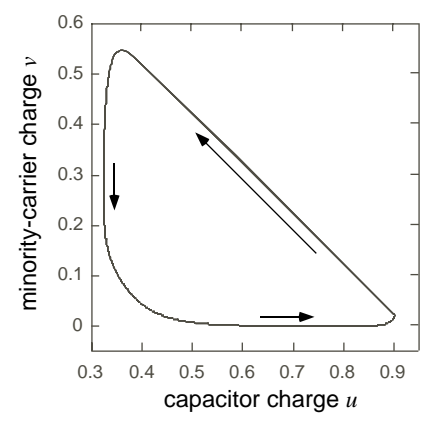

(b) limit-cycle attractor
Fig. 3. Dynamical properties of the oscillatory reaction cell (simulation). (a) Time evolution; (b) limit-cycle attractor on the $u-v$ plane.

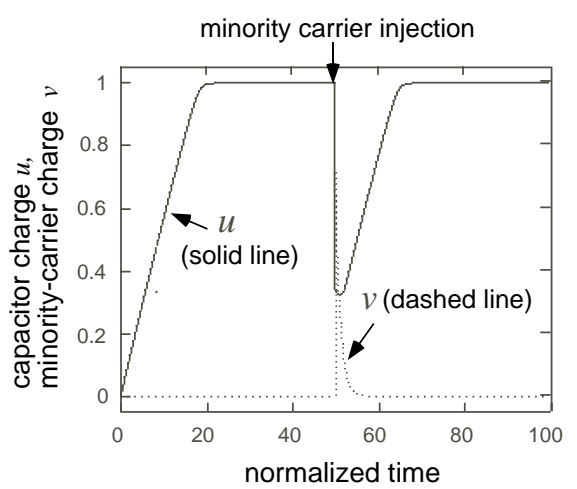

Fig. 4. Time evolution in the excitatory reaction cell (simulation).

of the reaction cell. The cell settles down in the stable state of $u=1$ and $v=0$, and no further charge occurs as it stands. In the simulation, minority carriers were injected from the outside at time $=50$. Triggered by this injection, the $p-n-p-n$ diode turned on for once, and then returned to the stable state.

\section{Simulation Results}

We designed two-dimensional RD device by arranging the reaction cells on a plane and confirmed the device operation by computer simulation. At the position of each reaction cell, we used the following RD equations that describe spatiotemporal dynamics of the cell:

$$
\begin{aligned}
& \frac{\partial u(x, y)}{\partial t}=i(u)-\frac{u}{\tau(u, v)} \\
& \frac{\partial v(x, y)}{\partial t}=D_{v} \nabla^{2} v-v+\frac{u}{\tau(u, v)}
\end{aligned}
$$

where $(x, y)$ are the rectangular coordinates of any point on the RD-device plane, $\nabla^{2}$ the Laplacian operator, and $D_{v}$ the normalized diffusion coefficient. In other positions where no reaction cell exists, we used the following equations:

$$
\frac{\partial u(x, y)}{\partial t}=0, \quad \frac{\partial v(x, y)}{\partial t}=D_{v} \nabla^{2} v-v .
$$

We solved these equations numerically using the conventional FDTD method.

Figure 5 shows a result for a device with $200 \times 200$ excitatory reaction cells. The spatial density of minority carriers is represented in grayscale ( $v=0$ : black, $v=1$ : white). With periodic
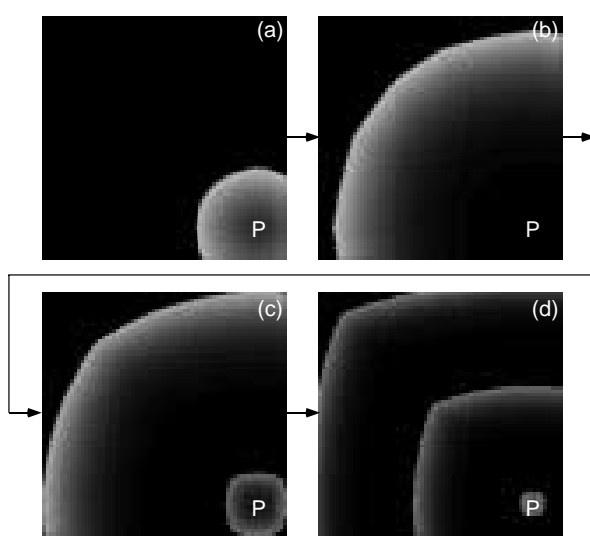

Fig. 5. Generation of spreading concentric patterns in a RD device (simulation).
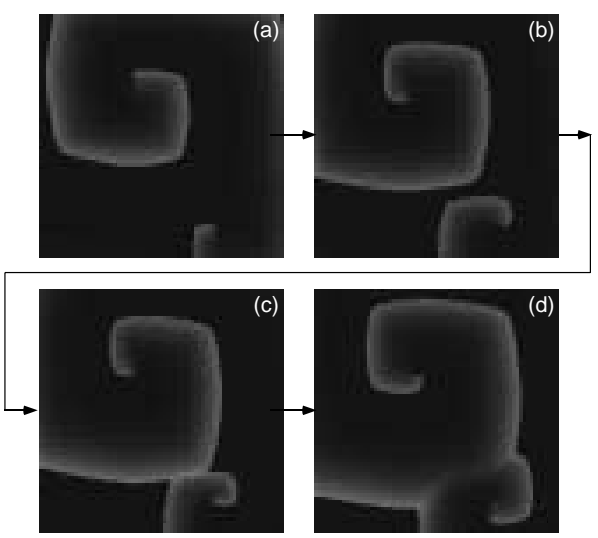

Fig. 6. Generation of rotating spiral patterns in a RD device (simulation).

injection of minority carriers at a point ( $\mathrm{P}$ in Fig. 5), the RD device produced spreading concentric waves of minority carriers. This results indicate that the injected carriers diffused around the injection point and successfully induced a chain of reactions of the cells.

Figure 6 shows a result of the excitatory RD device without external injections of minority carriers. With an appropriate initial pattern of minority-carrier densities, the RD device produced rotating spiral patterns of minority carriers. Notice that the wave disappears at collision points [Figs. 6(c) through (d)] because of the depletion of minority carriers. This is the same phenomenon as observed in natural RD systems.

\section{SUMMARY}

We proposed a novel silicon device for imitating autocatalytic and dissipative phenomena of the reaction-diffusion (RD) systems. Numerical simulations showed that the proposed RD device can successfully produce concentric and spiral waves in the same way as natural RD systems. Our results indicate that the proposed RD device will be an useful tool for developing novel hardware based on the RD mechanism.

\section{REFERENCES}

[1] G. Nicolis and I. Prigogine, Self-organization in Nonequilibrium Systems - From Dissipative Structures to Order through Fluctuations. John Wiley \& Sons, Inc., 1977. 